\title{
Design of Coreless printed circuit board (PCB) Step-Down Transformer -An Assessment of the Optimal Operation Frequency
}

\author{
Mohammadali Hashemi ${ }^{1}$, Ali Khalili Mobarakeh ${ }^{2}$,Shadi Mahmoodi \\ Khaniabadi ${ }^{3}$,Mohammad Ali Bagheri ${ }^{4}$, Mohd Fadzil Ain ${ }^{5}$ \\ 1, 2,3,4,5 School Of Electrical And Electronic Engineering, Engineering Campus, Universiti Sains Malaysia, \\ 14300 Nibong Tebal, Penang Malaysia
}

\begin{abstract}
Coreless PCB transformers are one of the most efficient transformers due to their zero core losses, low cost and simplicity of the fabrication. In this paper, a novel two layers PCB transformer is designed and simulated using CST Studio 3D software. In addition, since the optimal operating frequency has a profound impact on the efficiency of the circuit, this parameter is identified using simulation in this work.
\end{abstract}

Keywords - Coreless PCB transformer, maximum coupling frequency, coupling coefficient, resonant frequency

\section{INTRODUCTION}

For over a century, transformers have been used for electrical isolation and energy/signal transfer. In traditional transformers, ferrite cores have been employed to provide the magnetic path for signal and energy transfer. High manufacturing/labour cost, large size and high weight are serious challenges of manually-wound transformers and inductors. Skin and proximity effects, leakage inductance and unbalanced magnetic flux distribution are other problems involved in high frequency magnetic which generate localized hot spots and reduce the coupling coefficient. Recently, planar technology has been employed to reduce cost, weight, and size maintaining high power density (at least $24 \mathrm{~W} / \mathrm{cm}^{2}$ ). Coreless printed circuit board (PCB) transformers have the benefits of low cost, very high power density, no limitation due to magnetic cores, no magnetic loss and ease of manufacturing [1]. Owing to the low cost and simple transformer construction, a coreless PCB step-down transformer can be applied for industrial applications such as DC-DC converters for applications like WLAN Access-points, Power over Ethernet, IP phones, Switch Mode Power Supply (SMPS) and for a wide variety of telecom applications. This paper presents the design, simulation, and theory of coreless PCB step-down transformer.

\section{PROPOSED DESIGN}

In order to design coreless PCB, step down transformer have been used from three spiral windings which are etched on the two layers of the FR-4 PCB laminate with approximate breakdown voltage $50 \mathrm{kv} / \mathrm{mm}$. In this design, there are two primary windings (P1 and P2) in two different layers being connected by via hole and one secondary winding $(\mathrm{S})$ in between the primary windings on the opposite side of the one doubled-side PCB laminate aiming to minimize the leakage inductances. In [2], the inductive characteristics of coreless PCB transformers with different geometric parameters includes: i) outermost radius, ii) number of turns, iii) conductor width, iv) laminate thickness and v) conductor thickness are investigated. Figure1 shows the designed coreless PCB transformer. The geometrical parameters play an important role in operation of transformer. The electrical parameters such as resistance, capacitance and inductance depend on geometrical parameters. The dimensions of the coils, the PCB layout, the copper thickness and the track width must be selected with respect to the maximum allowable power losses [3].The geometrical parameters of multilayer coreless PCB step-down transformer are presented in table.

Table.1. Geometrical parameters of transformer

\begin{tabular}{|c|l|c|}
\hline S. No & Parameters & Value \\
\hline 1 & No. of primary/secondary turns & $20 / 10$ \\
\hline 2 & Track separation $[\mathrm{mm}]$ & 0.4 \\
\hline 3 & Width of conductor $[\mathrm{mm}]$ & 0.6 \\
\hline 4 & Outermost Diameter $[\mathrm{mm}]$ & 42 \\
\hline 5 & Height of conductor $[\mu \mathrm{m}]$ & 35 \\
\hline 6 & Board Thickness $[\mathrm{mm}]$ & 0.4 \\
\hline 7 & Air gap between two layers PCB $[\mathrm{mm}]$ & 0.4 \\
\hline
\end{tabular}




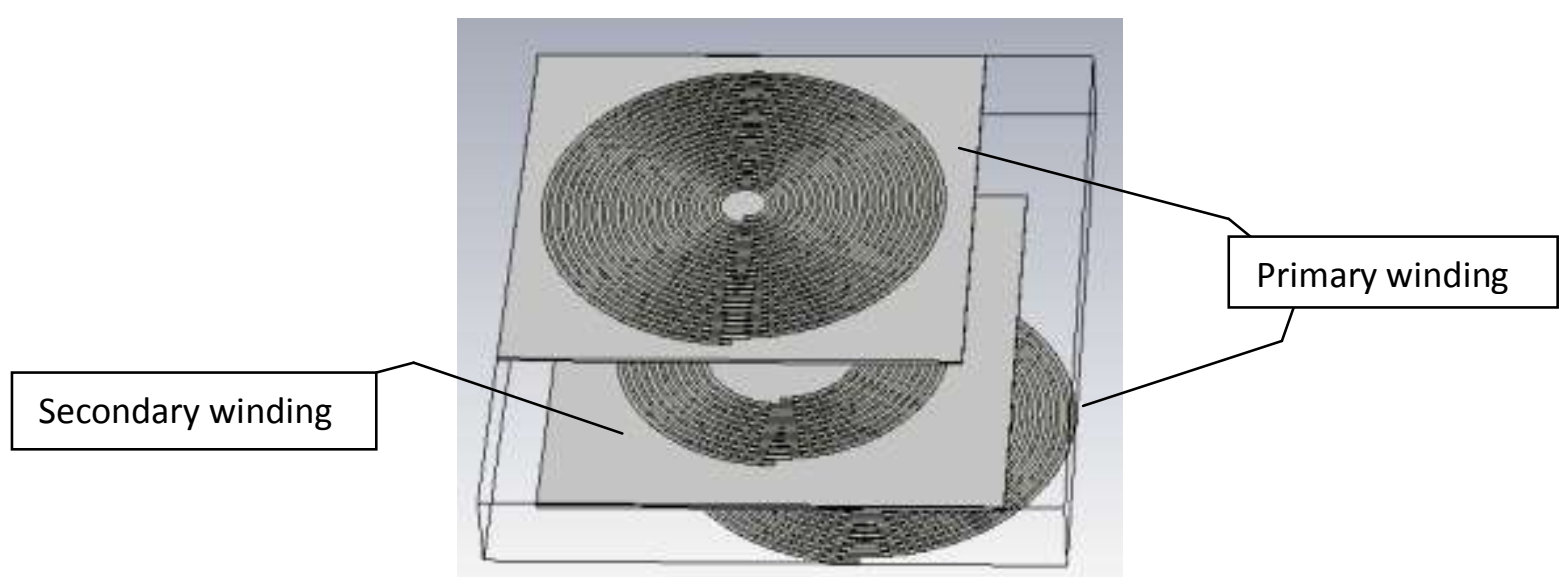

Figure 1.Three dimensional (3-D) structure of coreless PCB transformer

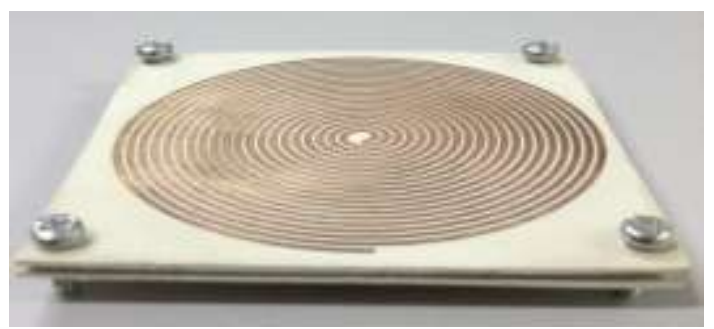

Figure 2. Prototype of Coreless PCB Step-down Transformer

\section{OPERATION Of Coreless PCB Transformer Parameters}

In order to evaluate parameters such as voltage gain, input impedance and resonant frequency in this kind of transformers we have used a high frequency equivalent circuit of transformer as it is presented in Fig 3. A parallel load across the secondary winding has been employed.



Figure 3. High frequency model of coreless PCB transformer

In this step, the electrical and initial parameters of coreless PCB step-down transformer have been calculated and measured at $100 \mathrm{KHz}$ by employing a LCR meter 4263B. The electrical parameters such as the leakage/self inductance of the primary ' $L_{l k p}{ }^{\prime} /{ }^{\prime} L_{P}$ ' is obtained by short and open circuiting the secondary winding respectively and vice versa. The precise initial parameters of transformers are gained by passing them into the high frequency model.

Table.2. Electrical Parameters of the Transformer

\begin{tabular}{|c|l|c|}
\hline S.NO & Parameters & Value \\
\hline 1 & Primary/Secondary self inductance $[\mu H]$ & $46.3 / 11.5$ \\
\hline 2 & Primary/Secondary leakage inductance $[\mu H]$ & $0.56 / 0.13$ \\
\hline 3 & Primary/Secondary Mutual inductance $[\mu H]$ & $42.1 / 9.5$ \\
\hline 4 & Primary/Secondary DC resistance $[\Omega]$ & $0.48 / 0.29$ \\
\hline 5 & Inter winding capacitance $[p F]$ & 21 \\
\hline
\end{tabular}

Regarding to high frequency model of transformer, the resonant frequency $\left(f_{0}\right)$, voltage gain $\left(\mathrm{V}_{\mathrm{S}} / \mathrm{V}_{\mathrm{P}}\right)$ and the input impedance $\left(Z_{\text {in }}\right)$ can be calculated as follows [4]-[5]-[6]: 
$f_{0}=\frac{1}{2 \pi \sqrt{L_{e q} C_{e q}}}$

where

$L_{\text {eq }}=L_{l k s}{ }^{\prime}+L_{m p}$ ?? $L_{l k p}$

Here, $L_{m p}$ and $L_{l k p}$ are mutual and primary leakage inductance respectively. And $L_{l k s^{t}}$ is secondary leakage inductance referred to the primary.

$C_{e q}=C_{r}{ }^{t}+C_{p s}{ }^{\prime}$

Where, $C_{r^{r}}$ is sum of the secondary winding capacitance and an externally connected capacitance referred to the primary and $C_{p S^{r}}$ Capacitance between primary and secondary winding referred to the primary. It determines that the resonant frequency can be limited by equivalent capacitance and inductance of the circuit model. The value of resonant capacitors across the secondary winding will play an important role in the bandwidth of the transformer. The intra-winding capacitances of transformer are very small and can be ignored according to [7].

$H(f)=\frac{V_{s}}{V_{p}}=\frac{\frac{1}{X_{1}}+j(2 \pi f) c_{p s} r Y 1}{n Y}$

$Z_{i n}=\frac{1}{s C_{p s}{ }^{g}\left(1-n \times \frac{V_{s}}{V_{p}}\right)+\frac{(1-A)}{X_{1}}+s C_{p p}{ }^{r}}$

Where ' $\mathrm{n}$ ' is number of turns

$R_{s}^{\prime}=n^{2} R_{s}$

$L_{l k s}{ }^{r}=n^{2} L_{l k s}$

$C_{P P}{ }^{\prime}=C_{P P}+\frac{n-1}{n} C_{p s}$

$C_{r}^{\prime}=\frac{1}{n^{2}} C_{r}+\frac{1-n}{n} C_{p s}$

$C_{p s},=\frac{1}{n} C_{p s}$

$X_{l}=R_{P}+s L_{l k p}$

$X_{2}=R_{s}{ }^{\prime}+s L_{l k s}{ }^{\prime}$

$Y_{l}=X_{2}\left[\frac{1}{X 1}+\frac{1}{s L_{m p}}\right]+1$

$Y_{2}=\frac{1}{X 2}+s C_{p s}{ }^{\prime}+s C_{r}{ }^{\prime}+\frac{1}{n^{2} R_{L}}$

$Y=-\frac{1}{X_{2}}+Y_{1} Y_{2}$

$A=\frac{s C_{p s}{ }^{s}+\frac{X_{z}}{X_{1}} Y_{2}}{Y}$

The coupling coefficient is given by [ $[\underline{8}]$

$K=\frac{L_{m}}{\sqrt{L_{p} L_{g}}}$

$L_{m p}=L_{p}-L_{l k p}$

$L_{m s}=L_{s}-L_{l k s}$

$L_{m}=\sqrt{L_{m p}-L_{l k s}}$

Where, $L_{m}$ is the mutual inductance between the primary and secondary winding and ' $L_{p}$ ' and ' $L_{g}$ ' are primary and secondary self inductance of winding.

In order to increase the input impedance, voltage gain, and the energy efficiency of the transformer, a resonant capacitor across the secondary winding has been employed.

\section{ASSESSING OPTIMAL OPERATING FREQUENCY}

In this section, frequency of maximum coupling, input impedance and phase of a coreless PCB stepdown transformer for operating at or near this frequency has been investigated. Finding the optimal operating frequency, results in having a better assessment, and prediction of the transformer's bandwidth. Same 
parameters as shown in Table 1 are used for simulation in this section. A parallel RC load is coupled on secondary winding for simulating. In addition, the transformer is tested within the frequency range of 10KHZ$100 \mathrm{MHz}$. The simulated maximum coupling input impedance and phase angle using CST Microwave Studio are plotted in Fig 4 to Fig 6. Fig 4, shows that the maximum coupling frequency (MCF) occurs at 17.848MHz. From Fig 5, it can be seen that the maximum impedance frequency (MIF) occurs at $17.848 \mathrm{MHz}$ and phase angle is placed between 18 and $20.3 \mathrm{MHz}$.

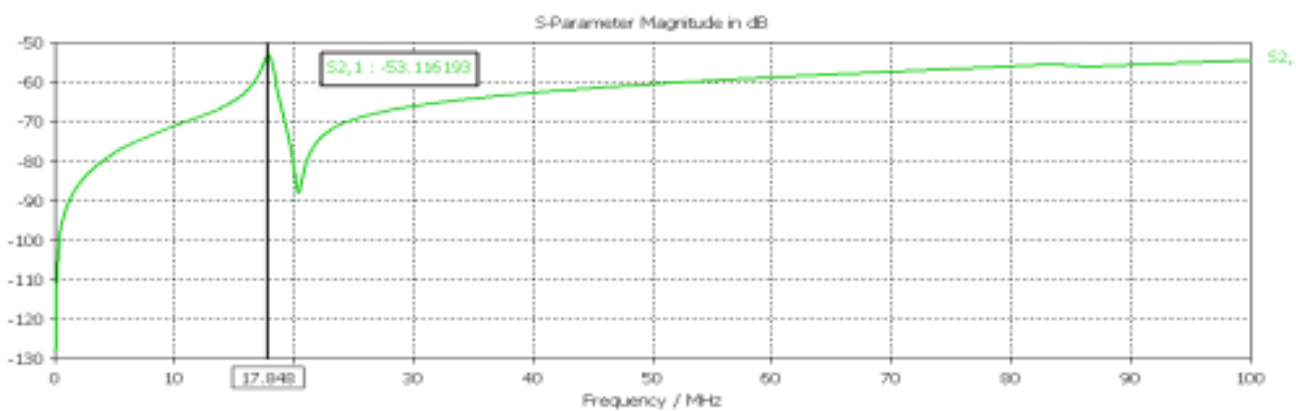

Figure. 4. The maximum coupling frequency (MCF) with $\mathrm{C}_{\mathrm{r}}=12 \mathrm{nF}$ and $\mathrm{R}_{\mathrm{L}}=100 \Omega$

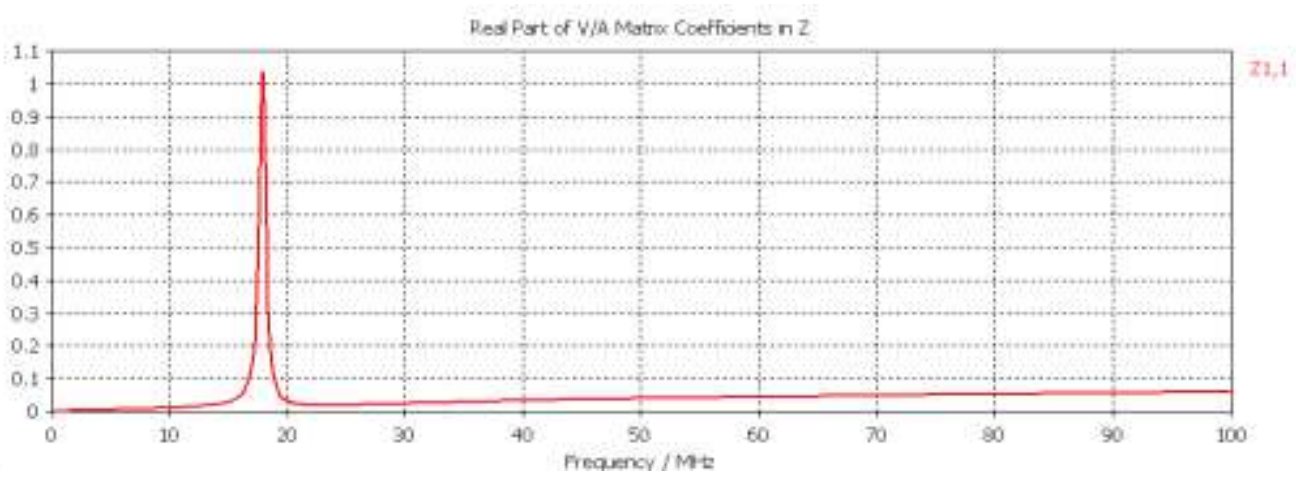

Figure. 5. Simulated result of input impedance of the transformer at $\mathrm{C}_{\mathrm{r}}=12 \mathrm{nF}$ and $\mathrm{R}_{\mathrm{L}}=100 \Omega$

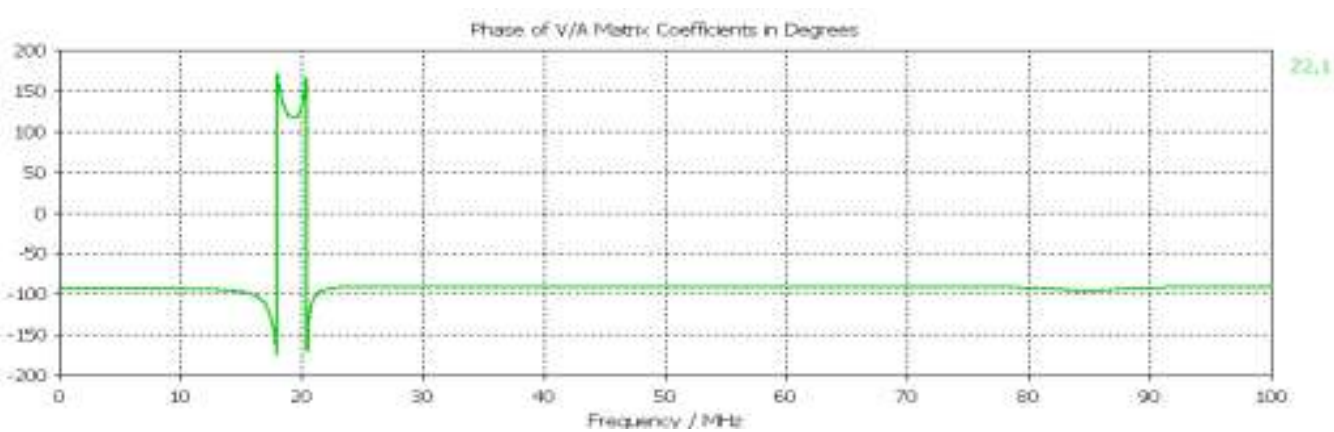

Figure 6. Simulated results of the phase angle of transformer at $C_{r}=12 n F$ and $R_{L}=100 \Omega$

Fig. 5 shows the values of maximum coupling frequency for different load capacitance with a constant load resistance $\left(\mathrm{R}_{\mathrm{L}}=100 \Omega\right)$.

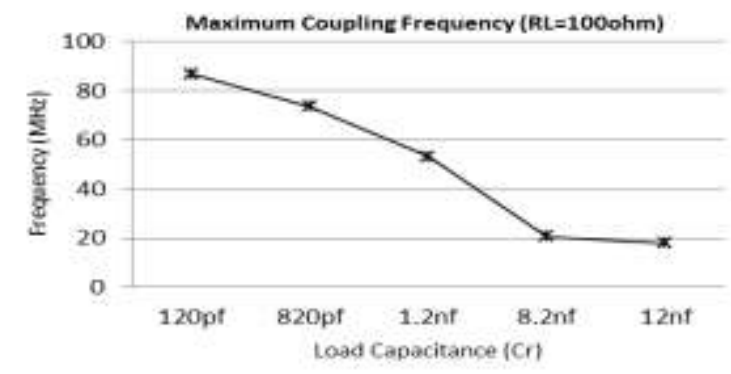

Figure 7. The values of maximum coupling frequency at different load capacitance 
In order to minimize the input current and as a result, the power dissipated at the gate drive circuits, the operating frequency of the transformer should be near or at the high frequency end of the usable operating region. It also shows the output capacitance plays significant role in the bandwidth of the transformer.

\section{CONCLUSION}

In this paper the design, simulation and analysis of a novel coreless PCB step-down transformer were presented. The simulation results were used to evaluate the maximum coupling and the optimal operating frequency of the proposed transformer. We found that the maximum coupling frequency is almost $17.8 \mathrm{MHz}$. In addition, the obtained results showed that the load capacitance across the secondary winding has an important effect on the maximum coupling frequency.

\section{Acknowledgment}

We would like to express the great gratitude to our supervisor Associate Professor Dr. Mohd Fadzil Ain for his excellent guidance and providing us with an excellent atmosphere for doing research at Universiti Sains Malaysia.

\section{REFERENCES}

[1] S. Y. R. H. a. H. S.-H. C. S.C.Tang, "Characterization of Coreless Printed Circuit Board (PCB) Transformers-A Fundamental Concept for Signal and Energy Transfer," IEEE Transactions on Power Electronics, vol. 15, 2000.

[2] S. Y. R. H. S.C.Tang, and Henry Shu-Hung Chung, "Characterization of Coreless Printed Circuit Board (PCB) Transformers," IEEE, vol. 15, NOVEMBER 2000

[3] P. K. S. R.Steiner, F.Krismer and J.W.Kolar, "Contactless Energy Transmission for an Isolated 100W Gate Drive Supply Of a Medium Voltage Converter," IEEE, 2009.

[4] H. B. K. a. D. K. B. Radhika Ambatipudi, "Coreless Printed Circuit Board (PCB) Step-down Transformer for DC-DC Converter Applications," World Academy of Science, Engineering and Technology 70 2010, 2010.

[5] H. S.-H. C. S.Y.(Ron) Hui, and S.C. Tang, "Coreless Printed Circuit Board (PCB) Transformers for Power MOSFET/IGBT Gate Drive Circuits," IEEE TRANSACTIONS ON POWER ELECTRONICS, vol. 14, MAY 1999.

[6] S. C. T. S. Y. (Ron) Hui, and Henry Shu-Hung Chung,, "Optimal Operation of Coreless PCB Transformer-Isolated Gate Drive Circuits with Wide Switching Frequency Range," IEEE TRANSACTIONS ON POWER ELECTRONICS, vol. 14, MAY 1999.

[7] R. A. Hari Babu Kotte, Kent Bertilsson, "A ZVS Flyback DC-DC Converter using Multilayered Coreless Printed-Circuit Board (PCB) Step-down Power Transformer," Proceedings of World Academy of Science, Engineering and Technology, vol. 70, pp. 148$155,2010$.

[8] R. A. a. K. B. Hari Babu Kotte, "High Speed Cascode Flyback Converter Using Multilayered Coreless Printed Circuit Board (PCB) Step-Down Power Transformer," $8^{\text {th }}$ International Conference on Power Electronics-ECCE Asia May 30-June 3,2011, The Shilla Jeju, Korea, 2011. 